\title{
M-learning y realidad aumentada, tecnologías integradas para apoyar la enseñanza del cálculo
}

\author{
Luis Eduardo Pedraza ${ }^{1}$ \\ Valbuena SD ${ }^{2}$
}

\begin{abstract}
Resumen
Los dispositivos móviles son cada vez más comunes en nuestra sociedad, gracias a que hoy en día a través de estos es posible realizar distintas tareas como: revisar correos electronicos, leer periódicos, consultar el estado del tiempo, entre otras.

En el presente artículo se presenta el desarrollo de una plataforma móvil, con realidad aumentada, para apoyar los procesos de enseñanza y aprendizaje del cálculo. Esta permite solucionar ejercicios tales como: límites, derivadas e integrales, consultar los contenidos de las asignaturas de cálculo diferencial, integral y vectorial, ilustrados con ejemplos y ejercicios propuestos. Además, se incorporan contenidos multimedia utilizando realidad aumentada, con los que se pueden apreciar representaciones gráficas de funciones tridimensionales.

En un futuro muy cercano la plataforma móvil con realidad aumentada puede ser utilizada como herramienta tecnológica para contribuir en la comprensión de los conceptos expuestos en las asignaturas de cálculo.
\end{abstract}

Palabras clave: cálculo, herramienta tecnológica, m-learning, proceso enseñanza-aprendizaje, realidad aumentada. 


\title{
M-learning and augmented reality, built to support the teaching of computing technologies
}

\begin{abstract}
The mobile devices are more commons in our society, because today across this is possible to make several works such as: check emails, read journals, check the weather, etc.

In this article presents the development of a mobile platform whit augmented reality for the support the learning and teaching process of the calculus. This, it allows solving exercises such as: limits, derivates and integrals, consulting the contents of the subject of calculus: diferential, integral and several variables illustrated with examples and exercises proposed. Also, incorporate multimedia contents using augmented reality, with this is possible appreciate graphics representations of tridimensional functions.

In a nearby future the mobile platform whit augmented reality can be used such as technological tool for the contribution in the understanding of concepts in the subject of calculus.
\end{abstract}

Keywords: Augmented reality, calculus, teaching-learing process, tecnological tool, M-learning.

Recibido: 05/08/2014

Aceptado: 13/11/2014

\section{Introducción}

Un estudio realizado por la Universitat Ramon Llull (Pere, 2012), demuestra que la aplicación adecuada de las tecnologías de la información y las comunicaciones (TIC), en el aula, puede ser una herramienta efectiva para mejorar significativamente los resultados académicos de los estudiantes. Resaltando que no basta dotar las aulas con la tecnología sino que deben tenerse contenidos educativos adecuados y profesorado con la formación necesaria para aprovechar el valor añadido de las TIC.

El avance en las TIC ha permitido explorar nuevas metodologías de enseñanza, tales como el e-learning y el m-learning, buscando reducir las limitaciones de tiempo y espacio en la formación académica de los estudiantes y fortalecer los procesos de enseñanza y aprendizaje. 
El m-learning son ambientes de aprendizaje basados en la tecnología móvil, destinados a mejorar e impulsar los procesos de enseñanza y aprendizaje. Esto se logra integrando el e-learning (sistemas de enseñanza y aprendizaje a través de redes digitales), con los dispositivos móviles de comunicación con la finalidad de producir experiencias educativas en cualquier lugar y momento.

Por otra parte, la realidad aumentada es una variación de los Ambientes Virtuales (AV), o Realidad Virtual (RV), como se conoce más comúnmente. La Realidad Virtual sumerge al usuario dentro de un ambiente sintético (generado por la computadora); mientras está inmerso, el usuario no puede ver el mundo real alrededor de él. En contraste, la Realidad Aumentada le permite al usuario ver el mundo real, con objetos virtuales sobrepuestos sobre el mundo real, o compuestos con él (Azuma, 1997).

Combinar el m-learning con la realidad aumentada, es una posibilidad que se abre para explorar nuevos enfoques y metodologías para la optimización y el fortalecimiento de la enseñanza y el aprendizaje de las matemáticas. Además de realizar aportes externos a los contenidos expuestos en el aula de clase.

Trifonova (2003), muestra en su revisión bibliográfica sobre el m-learning varias aplicaciones en las que se destaca la generación de contenido para el estudio y la enseñanza utilizando smartphones y el protocolo de comunicaciones inalámbricas para dispositivos móviles. Generalmente se busca que los contenidos en m-learning sean adaptables y que potencien al estudiante desde el punto de vista pedagógico y cognitivo.

Esteban, Restrepo, Trefftz, Jaramillo y Álvarez (2012), desarrollan una herramienta de realidad aumentada, que les permitió el diseño de experiencias de aprendizaje significativas para los estudiantes y les ayudó a potenciar la comprensión de los conceptos objeto de estudio. Esto generó que los estudiantes repasaran, pensaran e integraran los conceptos vistos en otras tutorías o eran capaces de intuir conceptos a desarrollar en tutorías posteriores.

Fitzgerald, Adams, Ferguson, Gaved, Mor y Thomas (2012a), destacan que el uso de la realidad aumentada en la educación, y particularmente el m-learning, se encuentra aún en estudio y las nuevas contribuciones son vistas como buenas y útiles para crear experiencias de aprendizaje efectivas.

Es por esto que en este trabajo se plantea el desarrollo de una plataforma móvil, con realidad aumentada como herramienta tecnológica para apoyar los procesos de aprendizaje y enseñanza de las asignaturas que son catalogadas complejas por la mayoría de estudiantes de ingeniería. Además de esto, se espera contribuir a la comunidad académica y científica con una nueva experiencia al integrar el m-learning y la realidad aumentada como herramientas tecnológicas de apoyo al aula de clase. 


\section{Realidad Aumentada - RA}

La realidad aumentada es una inserción de objetos virtuales en un ambiente real con el auxilio de un dispositivo tecnológico. Esta tecnología hace que el individuo tenga una sensación de que los objetos existan en el ambiente (Biazus, 2012 ). Azuma (2001), reduce el énfasis sobre los objetos gráficos y establece tres propiedades fundamentales de la realidad aumentada que son: la combinación de objetos reales y virtuales en ambientes reales, un sistema que registre objetos virtuales y reales con cada otro, y la ejecución interactiva en tiempo real.

Esta tecnología, aun en crecimiento e investigación, ha sido aplicada en múltiples áreas; por ejemplo, Feiner, MacIntyre, Höllerer y Webster (1997), describen el desarrollo de un prototipo la superposición de gráficos en $3 \mathrm{D}$ de la realidad aumentada con las características de la computación móvil. En la aplicación se presenta información sobre el campus universitario, utilizando head-tracked, head-worn e información en 3D.

En el desarrollo de aplicaciones con RA para la educación, se encuentra Construct3D que es una herramienta de construcción geométrica tridimensional, diseñada específicamente para la educación de la matemática y la geometría; está basada en un sistema móvil colaborativo con realidad aumentada.

La realidad aumentada se presenta también como una estrategia de visualización para el cálculo de varias variables ya que permite visualizar objetos creados a partir de ecuaciones con los que es posible interactuar de manera semejante a como lo haría un estudiante con modelos reales (Esteban, Restrepo, Trefftz, 2009).

Esteban, Restrepo, Trefftz, Jaramillo y Álvarez (2012), describen el desarrollo de un prototipo de Realidad Aumentada para la enseñanza del Cálculo en Varias Variables, que permite la visualización de conceptos matemáticos a partir de la creación de un objeto virtual, que se puede comparar con objetos reales, potenciando las posibilidades de comprensión de los conceptos matemáticos estudiados. La instrucción impartida se fundamentó en la pedagogía de la Enseñanza para la Comprensión, que propicia en los alumnos la integración de conceptos previamente adquiridos con los nuevos que son objeto de estudio y su utilización en forma creativa para solucionar diversas situaciones de su entorno.

Leitao, Brito y Rodrigues (2012), asumen que el contexto de enseñanza es propicio para la experimentación de nuevos modelos de enseñanza-aprendizaje, y presentan una aplicación educativa en el área de la geometría con el objetivo de potencializar la transferencia de conocimiento.

Gracias a los avances en los conceptos pedagógicos, las aplicaciones y la tecnología, y a la disminución en los costos de hardware, el uso de aplicaciones 
móviles con realidad aumentada puede llegar a ser factible, en un futuro muy cercano, para las instituciones educativas (Kaufmann, 2003).

\section{Retos pedagógicos}

La realidad aumentada es una tecnología en crecimiento y desarrollo, y por tanto se enfrenta a muchos retos en las áreas de aplicación para la que se necesite. En la parte pedagógica, la RA presenta varias cuestiones en común con otras tecnologías utilizadas para la enseñanza y el aprendizaje.

Lo novedoso de la tecnología puede disminuir las experiencias de aprendizaje, esto debido a que los estudiantes podrían estar más enfocados en sus nuevos dispositivos que en el cumplimiento de sus objetivos de aprendizaje (Fitzgerald, Adams, Ferguson, Gaved, Mor y Thomas, 2012b). Hay que tener en cuenta que muchos estudiantes no cuentan con dispositivos móviles capaces de soportar la RA; sin embargo, el número de personas que adoptan los smartphones continúa con un crecimiento bastante alto.

Desde el punto de vista de la enseñanza, se considera que primero se debe tener en cuenta los objetivos y metas que un tutor o educador quiere que sus estudiantes alcancen, antes de considerar que es lo mejor para lograr esto. En este aspecto la realidad aumentada puede no ser el mejor método a emplear respecto a otras tecnologías, que pueden ser más baratas, más robustas y mucho más apropiadas para las actividades de aprendizaje requeridas (Fitzgerald, Adams, Ferguson, Gaved, Mor y Thomas, 2012c).

\section{Mobile Learning}

Mobile learning es un nuevo paradigma de aprendizaje centrado, que cree realmente en el aprendizaje disponible en cualquier lugar y en cualquier tiempo, con sus diferentes características de los conceptos previos de aprendizaje como el aprendizaje basado en computador o el aprendizaje electrónico (Son, Lee y Park, 2004) . Este ha ayudado en la transmisión de materiales de aprendizaje a los estudiantes en una variedad de situaciones. Recolectando y enviando datos desde el trabajo, o simplemente comunicándose con los profesores y otros expertos fuera del aula de clase tradicional, el m-learning está rompiendo los límites del aprendizaje basado en la escuela tradicional (Bhargava, 2011).

El aprendizaje móvil puede ser considerado como una forma extrema de aprendizaje flexible, el cual ofrece a los estudiantes facilidades que permiten desarrollar un ambiente de aprendizaje más flexible (Seppälä, Alamäki, 2003). Kynäslahti (2003), identifica tres elementos para la movilidad que son valiosos para los profesores y estudiantes en sus procesos de aprendizaje y enseñanza, que son: conveniencia, oportunidad e inmediación. 
Entre las ventajas que se pueden encontrar al utilizar m-learning, se destacan:

a) Uso eficiente del tiempo: se habla de la utilización de los tiempos muertos, que son aquellos que se destinan en labores de transporte, espera para ser atendido, para ser empleados con fines de aprendizaje.

b) Expansión de la alfabetización digital: muchos de los usuarios de la tecnología móvil utilizan esta herramienta como medio de entretención y comunicación social, y aunque su uso como forma de aprendizaje no está muy difundido se considera de enorme potencial.

c) Accesibilidad: los dispositivos están al alcance de casi cualquier persona, así como los servicios necesarios para su uso.

d) Contacto social: el estudiante puede estar en contacto con compañeros y tutores en cualquier momento para recibir información y facilitar su aprendizaje.

e) Mejoramiento de la productividad: al hacer uso efectivo de tiempos muertos y el poder recibir retroalimentación a pedido, permite que los procesos de enseñanza- aprendizaje se hagan más rápido y de modo efectivo, aumentando la productividad del estudiante.

f) Aprendizaje colaborativo: el estar en contacto con los compañeros de curso redunda en beneficio al momento de realizar trabajos que involucren colaboración, ya que la misma se refuerza con la presencia digital de los integrantes permitiendo aportes y retroalimentación.

g) Incremento en el estudio individual: los aparatos usados en este tipo de tecnología son personales y por tanto su uso implica constancia, responsabilidad y motivación por parte del estudiante para cumplir con sus deberes y trabajos.

h) Información eficaz: los contenidos transmitidos a los estudiantes por este medio deben tener características especiales y una de ellas es su tamaño (Lee, 2005), nadie va a pasar horas leyendo en un celular o PDA. La información enviada deber ser sintética y completa.

i) Los profesores pueden diseñar y poner a disposición de los alumnos materiales que contribuyan al aprendizaje de sus alumnos, pudiendo estos, disponer de dichos materiales de manera asíncrona (Zambrano, 2009). 


\section{Resultados}

Para el desarrollo de la plataforma, se utilizó una metodología basada en fases secuenciales que permitieron lograr el objetivo del proyecto.

Inicialmente se exploraron los contenidos programáticos de las asignaturas de cálculo diferencial, integral y vectorial de la Universidad Nacional, Universidad de los Andes, Universidad Eafit y de la Universidad de la Costa. De los conjuntos de temas de cada una de las asignaturas, en las universidades exploradas, se realizó la intersección de estos para consolidar los contenidos digitales a ser implementados en la plataforma.

Posterior a esto seleccionaron las herramientas informáticas a utilizar, derivados de una comparación entre varias de ellas, y se estableció la arquitectura a utilizar en la plataforma.

Las plataformas seleccionadas fueron: Android, por ser open source, por brindar una amplia gama de servicios disponibles, y gran calidad en gráficos y sonido. Además de esto es una tecnología que sigue en continuo desarrollo y cuenta con una gran comunidad de usuarios que van en aumento.

Blender, es el software utilizado para la construcción de los objetos tridimensionales que serán utilizados en el desarrollo de la plataforma. Es open source y permite la exportación a diferentes formatos tales como: obj, requerido para posteriormente ser utilizados por vuforia, y las diferentes características de los objetos.

Sencha, es un framework para desarrollar aplicaciones móviles ricas en interfaz gráfica de usuario, open source, cuenta con una gran comunidad de soporte y una documentación detallada.

Phonegap, es un framework utilizado para el desarrollo de aplicaciones móviles a partir de una aplicación web, es open source y permite la integración con Sencha.

Vuforia, es un framework para desarrollar aplicaciones con realidad aumentada en dispositivos móviles, es open source, soporta marcadores naturales, tiene una documentación bastante completa, es un proyecto que continua en desarrollo gracias a la gran comunidad de desarrolladores con la que cuenta. La arquitectura utilizada para la plataforma es cliente-servidor, siendo el cliente los dispositivos móviles y el servidor un ordenador en el que se encuentran almacenados los contenidos digitales en una base de datos y se accede a estos a través de un web service desarrollado en php y desplegada en un web server apache. 
M-learning y realidad aumentada, tecnologías integradas para apoyar la enseñanza del cálculo. Artículo producto de la investigación.

Finalmente se obtiene la plataforma que se ejecuta sobre el sistema operativo Android y que permite solucionar variados ejercicios de aplicaciones del cálculo, tales como: límites, derivadas e integrales.

Para esto la aplicación realiza una petición Ajax a un servicio web que provee Wolfram alpha, el cual retorna un texto en formato xml con la solución del ejercicio que es interpretado y procesado por la aplicación para luego ser presentado al usuario.

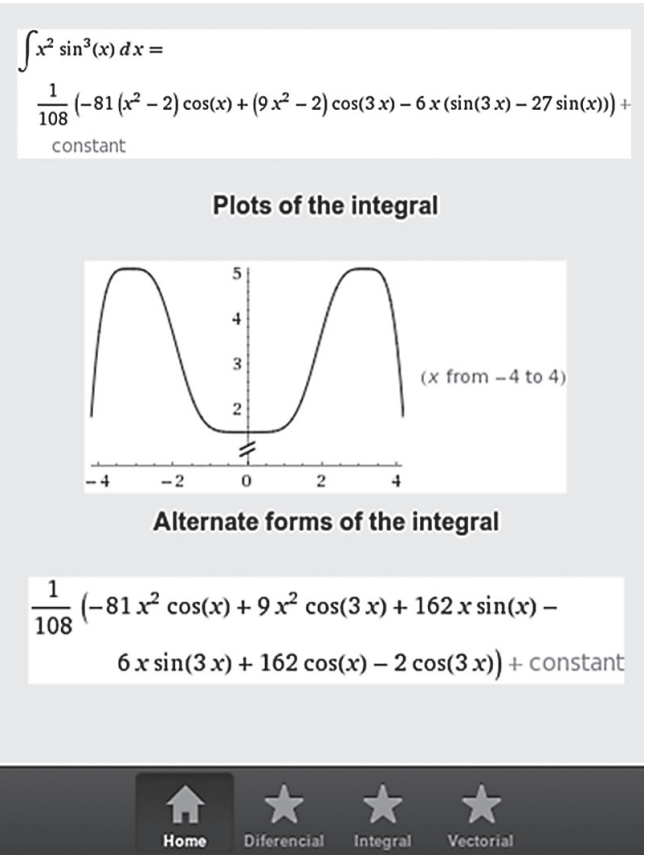

Figura 1. Solución de la integral

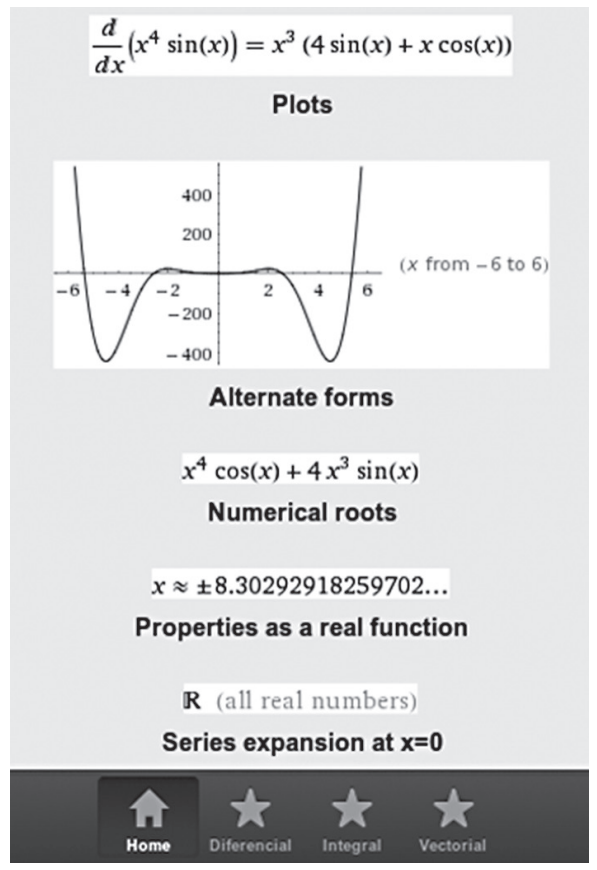

Figura 2. Solución de la derivada

$$
\frac{d\left[x^{4} \operatorname{sen}(x)\right]}{d x}
$$

Además de esto, la plataforma cuenta con un menú en el que se puede acceder a cada uno de los temas de cada una de las asignaturas de cálculo diferencial, integral y vectorial. Esto se realiza con una petición Ajax al servidor, el cual ejecuta una consulta a la base de datos del tema solicitado y retorna una respuesta en formato Json para ser procesada y mostrada al usuario. 


\begin{tabular}{|c|c|c|}
\hline \multicolumn{3}{|c|}{ Diferencial } \\
\hline \multicolumn{3}{|c|}{ Funcion, Dominio y Rango } \\
\hline \multicolumn{3}{|c|}{ Notacion de funciones } \\
\hline \multicolumn{3}{|c|}{ Funciones definidas por más de una ecuación } \\
\hline \multicolumn{3}{|c|}{ Grafica de funciones } \\
\hline \multicolumn{3}{|c|}{ Intersección con los ejes } \\
\hline \multicolumn{3}{|c|}{ Problemas con funciones } \\
\hline \multicolumn{3}{|c|}{ Definición de limite } \\
\hline \multicolumn{3}{|c|}{ Estimación númerica de lmite } \\
\hline$\widehat{11}$ & और & और \\
\hline
\end{tabular}

Figura 3. Contenidos de la asignatura de cálculo diferencial en la plataforma.

\section{Magnitudes escalares}

Denominamos Magnitudes Escalares a aquellas en las que las medidas quedan plenamente expresadas por medio de un número y la correspondiente unidad. Ejemplo de ello son: Masa, Temperatura, Presión, Densidad, entre otras.

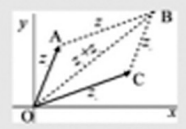

Ejemplo de representación gráfica de magnitudes escalares en el plano cartesiano.

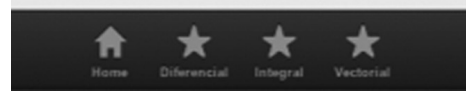

Figura 4. Información mostrada en la plataforma sobre el tema magnitudes escalares.

Por otra parte, el modulo de realidad aumentada permite a través de un image target, que es la definición formal de una función en el espacio, representarla gráficamente. Para esto se utiliza el framework vuforia, el cual realiza un reconocimiento de la imagen y a partir de esta se construye la representación gráfica.

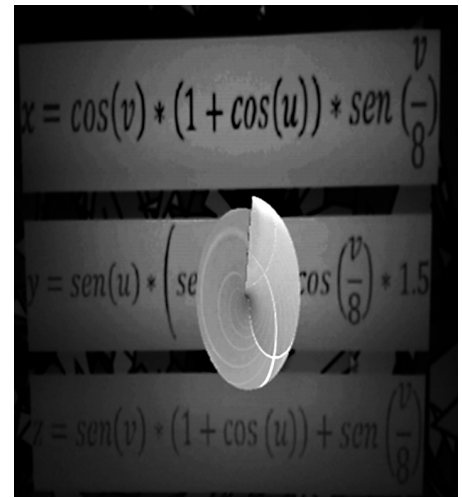

Figura 5. Representación de una función paramétrica en el espacio, utilizando realidad aumentada.

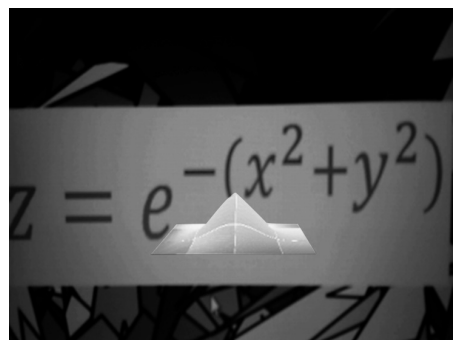

Figura 6. Representación de la función

$$
z=e^{-\left(x^{2}+y^{2}\right)}
$$

utilizando realidad aumentada.

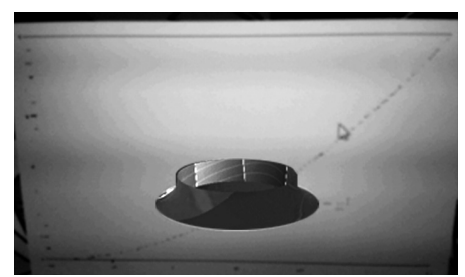

Figura 7. Representación del sólido revolución obtenido a partir de las funciones

$$
y=x^{2}, y=0, x=0, x=3
$$




\section{Agradecimientos}

El equipo de desarrollo del proyecto agradece a la Universidad de la Costa por prestar el ambiente de trabajo y laboratorios, y al Departamento Administrativo de Ciencia, Tecnología e Innovación - Colciencias por su apoyo económico.

\section{Conclusiones}

La realidad aumentada, utilizada como material didáctico complementario, puede contribuir a mejorar la comprensión de los conceptos estudiados en las asignaturas de cálculo diferencial, integral y vectorial.

La aplicación desarrollada, en un futuro muy cercano, puede ser utilizada como herramienta tecnológica para apoyar los procesos de enseñanza y aprendizaje de los cálculos diferencial, integral y vectorial.

El uso de tecnologías móviles enmarca una ventaja importante, ya que elimina las limitaciones de tiempo y espacio que se presentan en las metodologías de de enseñanza tradicionales.

\section{Referencias bibliográficas}

Bayonet, L., Patiño, A., Willmore, A. 2011. Realidad aumentada en el ámbito universitario. Santo Domingo: Universidad Iberoamericana.

Blender Foundation. s.f. Blender user manual, Disponible en: http://www.blender. org/.

Bover, A. 2010. Aplicación de gestión de información geolocalizada en Android. Cataluña: Universidad Politécnica de Cataluña, Facultad de Informática.

Esteban, P., Restrepo, J., Trefftz, H., 2009. Estrategias de visualización en el cálculo de varias variables. Revista Educación y Pedagogía. 18 (45): 121-131.

Esteban, P., Restrepo, J., Trefftz, H., Jaramillo, J.E., Álvarez, N. 2012. La realidad aumentada: un espacio para la comprensión de conceptos del cálculo en varias variables. Departamento de Ingeniería de Sistemas, Departamento de Ciencias Básicas, Medellín: Universidad Eafit.

Fabregat, R. 2011. Realidad aumentada al servicio del aprendizaje. Girona: Universidad de Girona. 
Fabregat, R., Gomez, S. 2011. Context-Aware Content Adaptation in m-Learning. Girona: Universidad de Girona.

Groner, L. 2011. ExtJS 4 First Look, Birmingham: Packt Publishing.

Hernández, R., Morales, M. 2012. Dispositivos móviles en la educación. Ciudad de Guatemala: Universidad Galileo.

Json. Introduciong json. Disponible en: http://www.json.org/.

Kaufmann, H., Schmalstieg, D. 2011. Mathematics and Geometry Education with Collaborative Augmented Reality. Interactive Media Systems Group, Vienna: Vienna University of Technology.

Leitão, R., Brito, A. y Rodrigues, J., 2012. A aplicação da Realidade Aumentada no ensino de sólidos geométricos: um projecto em desenvolvimento. 6th International Conference on Digital Arts - ARTECH 2012. Pp. 423-426. Faro: Portugal.

Mednieks, Z., Dormin, L., Meike, G., Nakamura, M. 2012. Programming Android, Second Edition. Sebastopol, CA: O’Reilly Media.

Nilsson, P., Sollervall, H. y Spikol, D. 2011. Mathematical Learning Processes Supported by Augmented Reality. Kalmar: Linnaeus University.

Sencha. 2012. Sencha Touch Documentation. Sencha .

Sood, R. 2012. Pro Android Augmented Reality. New York: Apress.

Vuforia. 2012. Vuforia Documentation. Qualcomm Austria Research Center .

Williams, G. 2012. Learn HTML5 and JavaScript for Android. New York: Apress.

Wolfram alpha, Bring computational knowledge to your web, mobile, desktop, and enterprise applications, Wolfram alpha api.

W3C. Extensible Markup Language (XML). Disponible en: http://www.w3.org/XML/.

Zambrano, J. 2009. Aprendizaje móvil (M-Learning). Inventum, 1 (7): 38-41. 